\title{
Determining the best warm ischemic time for patients undergoing partial nephrectomy for renal cancer
}

\author{
Greg Trottier, MD, PhD, FRCSC
}

See related article on page 40.

Cite as: Can Urol Assoc J 2011;5(1):44; D01:10.5489/cuaj.11015

$\mathrm{T}$ he experimental animal study on warm ischemia time (WIT) by Sabbagh and colleagues presents provocative and intriguing results. ${ }^{1}$ The strength of this study is the use of histologic outcomes as an endpoint for WIT. Using unilateral laparoscopic or open renal artery clamping for various time durations in a porcine model, the authors suggested that no permanent renal histopathologic damage occurred with up to 60 minutes of WIT in the clamped kidney. In addition, they demonstrated that up to 3 hours of pneumoperitoneum caused no histopathologic changes in the non-clamped, contralateral kidney 14 days after surgery. Should we extrapolate these results to humans and be comfortable with WITs of up to 60 minutes in patients undergoing partial nephrectomy for renal cancer?

Though the results of this study are attractive, they should be interpreted with caution for several reasons. ${ }^{2}$ Firstly, physiologic differences are noted between humans and animals, such as unique thrombinolysis kinetics in pigs, that could contribute to disparate WIT outcomes. ${ }^{3}$ Furthermore, although others have shown that there is little effect on early postoperative renal indicies with up to 90 minutes of WIT in pigs, ${ }^{3}$ studies examining the effects of WIT beyond 30 minutes in humans are conflicting. ${ }^{4}$ With regard to the reversible nature of the multifocal interstitial nephritis noted in all kidneys clamped for 5 to 60 minutes, the severity of nephritis was not quantified and the number of animals was low. Moreover, examination of renal tissue on postoperative day 14 may not reflect the true long-term histopatholo gic consequences of renal ischemia that may surface several months or years after surgery. Without quantitative and long term data, conclusions on whether clamping up to 60 minutes is safe based on early hisptopathology are limited.

As pointed out by the authors, renal artery clamping was performed without partial nephrectomy such that the effects of partial nephrectomy on WIT histopathologic outcomes could not be determined. ${ }^{1}$ The trauma of partial nephrectomy, the worse preoperative renal function in renal cancer patients and the comorbid diseases that commonly coincide with renal cancers could also affect WIT outcomes in the non-experimental setting. ${ }^{5}$ Lastly, longer duration of renal artery clamping (60 minutes) may cause renal artery scaring and stenosis that may not surface for months and could potentially manifest as hypertensive nephropathy years later.

Most of the unanswered questions regarding WIT are not easily controlled for in either experimental or clinical studies. Nonetheless, the results of this study are valuable and hypothesis-generating, but the translation to clinical practice will require human studies. Despite the lines of evidence promoting the safety of WITs beyond 30 minutes, logic dictates that renal artery clamp times should be minimized and balanced with safe tumour extirpation.

Urologic Oncology Fellow, University of Toronto, Toronto, ON

Competing interests: None declared.

This paper has been peer-reviewed.

\section{References}

1. Sabbagh R, Chawla A, Tisdale B, et al. Renal histopathology features according to various warm ischemia times in porcine laparoscopic and open surgery model. Can Urol Assoc J 2011;5:40-3; D01:10.5489/ cuaj.10010.

2. Simmons MN, Schreiber MJ, Gill IS. Surgical renal ischemia: a contemporary overview. J Urol 2008:180:19-30.

3. Laven BA, Orvieto MA, Chuang MS, et al. Renal tolerance to prolonged warm ischemia time in a laparoscopic versus open surgery porcine model. J Urol 2004;172:2471-4.

4. Becker F, Van Poppel H, Hakenberg OW, et al. Assessing the impact of ischaemia time during partial nephrectomy. Eur Urol 2009;56:625-34.

5. Huang WC, Levey AS, Serio AM, et al. Chronic kidney disease after nephrectomy in patients with renal cortical tumours: a retrospective cohort study. Lancet Oncol 2006; 7:735-40.

Correspondence: Dr. Greg Trottier, Division of Urology, Department of Surgical Oncology Princess Margaret Hospital, University of Toronto, Toronto, ON; 610 University Ave., Toronto, ON M5G 2M9; Greg.Trottier@uhn.on.ca 